\title{
Controversias sobre intuición, toma de decisiones económicas ${ }^{1}$
}

\author{
Controversies on intuition, economic decision making ${ }^{1}$
}

\author{
Luis Arturo Guerra Heredia ${ }^{2}$
}

\section{RESUMEN}

En la historia del pensamiento humano se ha dado importancia al estudio de la intuición, Platón, Descartes, Kant, etc. Han tomado posición al respecto, sin embargo, aún no está claro el concepto de intuición y se sigue dando un concepto idealista o metafísico. Recogiendo tesis de neurociencia descubrimos que la intuición no es irracional, es un conocimiento tácito que tiene que ver con la experiencia práctica e incluso se han descubierto células llamadas fusiformes, que podrían formar parte de ella. Por otro lado, a lo largo de muchos años se ha estudiado la toma de decisiones y en la teoría neo clásica se considera como uno de los postulados la racionalidad del consumidor, tesis que fue cuestionada por Kahneman y reforzada por Ariely, dándose más fuerza a las emociones en la toma de decisiones. Recogiendo tesis de la neurociencia, descubrimos que la toma de decisiones se da en torno a

\begin{abstract}
1 Aún no existe un entendimiento real sobre la intuición y de donde procede, al igual que existe un fuerte debate sobre si las decisiones que tomamos los humanos son racionales, emocionales o de otra índole, al respecto han opinado economistas, psicólogos, médicos y demás profesionales, sin embargo, siguen siendo temas complejos sobre los cuales hay mucho por investigar. A través de este artículo se busca contribuir al entendimiento de ambos conceptos y contribuir al debate de la relación de ambos aspectos tan importantes para la relación humana.

2 Economista, Universidad Nacional Mayor de San Marcos, Lima, Perú. Egresado de la maestría en Enseñanza Universitaria, Universidad Enrique Guzmán y Valle (CANTUTA), Lima, Perú. Maestrista en Neurociencias, Universidad Nacional Mayor de San Marcos, Lima, Perú. Actualmente labora en el Instituto de Ciencias y Humanidades, Lima, Perú. A. Óscar R. Benavides 2798, Lima, Perú. Correo Electrónico: aguerra@cbb.edu.pe
\end{abstract}

(C) Los autores. Este artículo es publicado por Pensamiento Crítico de la Facultad de Ciencias Económicas, Universidad Nacional Mayor de San Marcos. Este es un artículo de acceso abierto, distribuido bajo los términos de la licencia Creative Commons Atribucion - No Comercia_Compartir Igual 4.0 Internacional. (http:// creativecommons.org/licenses/by-nc-sa/4.0/) que permite el uso no comercial, distribución y reproducción en cualquier medio, siempre que la obra original sea debidamente citada. 
motivaciones, intereses y actitudes, las que a su vez responden a la tradición, cultura y economía de la persona. Así se descubre que no existe controversia entre racionalidad e intuición, y que la toma de decisiones considera la intuición de tal manera que toda decisión es producto en alguna medida de la intuición, aunque no siempre influye en ella.

Palabras Clave: Intuición; toma de decisiones; neocórtex prefrontal; plano epiconsciente; sistema conativo.

\section{ABSTRACT}

In the history of human thought, the study of intuition, Platon, Descartes, Kant, etc., has been given importance. They have taken a position on this, however, the concept of intuition is still not clear and an idealistic or metaphysical concept is still being given. Picking up neuroscience theses, we discover that intuition is not irrational, it is a tacit knowledge that has to do with practical experience, and even cells called fusiform, which could be part of it, have been discovered. On the other hand, decision making has been studied over many years, and in neo-classical theory, rationality of the consumer is considered as one of the postulates, a thesis that was questioned by Kannemann and reinforced by Ariely, giving more force to the emotions in making decisions. Collecting the thesis of neuroscience, we discover that decision-making takes place around motivations, interests and attitudes, which in turn respond to the person's tradition, culture and economy. Thus it is discovered that there is no controversy between rationality and intuition, and that decision-making considers intuition in such a way that every decision is a product of some measure of intuition, although it does not always influence it.

Keywords: Intuition; decision making; prefrontal neocortex; epiconscious plane; conative system.

\section{INTRODUCCIÓN}

Existen diferentes teorías respecto a la intuición, entre las que se le considera como algo mágico; otros como una especie de fuerza con la que nacemos y no la podemos incrementar ni disminuir, pero que si la dejamos de lado se inhibe; otros lo consideran como un don; etc. No se considerarán estas propuestas, solo estarán consideradas algunas de las principales propuestas científicas y documentadas dejando por tanto de lado las teorías mágicas o llamadas espirituales para establecer un rumbo de la intuición como actividad propia del hombre y exclusiva del ser humano.

En ese sentido se da por sentado y de hecho, que el sistema taxologico determinado por el botánico sueco Carlos Linneo en el siglo XVIII, que 
clasifico a todos los organismos de la Tierra, mediante la división en tres categorías específicas como: animal, vegetal y mineral, en la actualidad han sido ampliamente superadas, de igual manera es insuficiente la actual taxonomía según se cataloga al ser humano dentro del reino: animal; filum: Cordados; subfilo: Vertebrado; clase: mamífero; orden: primate; familia: homínido; tribu: hominini, genero: homo y especie: homo sapiens.

Es importante resaltar la tesis más coherente en donde se concibe al ser humano como ser particular y diferente, debido a que en su proceso de evolución ha ido transformando su medio y transformándose así mismo a tal magnitud que ya no forma parte del reino animal, al respecto Ortiz (1995, p. 2) "Hace pues varios miles de años que la especie Homo sapiens desapareció de la faz de la Tierra, justamente desde que la especie se convirtió en humanidad, y es por esta única razón que en los últimos miles de años cada uno de los hombres ya no es un animal, sino una personalidad".

Sobre la toma de decisiones se establece que los humanos tomamos decisiones todo el tiempo y en cada momento, incluso el no tomar decisión ya es una decisión por sí misma, aunque por cierto que la peor decisión que se puede tomar es no tomar decisiones. Decidimos desde que nos despertamos por las mañanas, incluso si nos levantamos en ese momento o no. Nuestras decisiones son entonces importantes no solo para el curso de nuestra existencia sino incluso para los que nos rodean porque influimos directa o indirectamente en nuestros allegados como nuestros padres, hermanos o hijos. Y más importante aún, son las decisiones económicas, porque vivimos en una sociedad creada por nosotros mismos, que tiene una estructura económica determinada y sobre la cual se erige toda una superestructura jurídica y política que determina el rumbo de nuestra sociedad. No existe una sola decisión económica que pueda considerase aislada o desarticulada del contexto social. Somos seres sociales por excelencia.

Al respecto Ortiz (2009, p.73) menciona que:

Desde los primeros cuchillos de piedra hasta las computadoras y las naves espaciales, como también los campos de cultivo y las granjas, las fábricas y las ciudades, todos son, en sentido lato, productos sociales. Por consiguiente, no debe llamar la atención que concluyamos en que los seres humanos transformados por la sociedad, que ya existe por sí misma, seamos, en sentido estricto, artificiales, aunque en este sentido 
preferimos llamarnos seres sociales, sistemas individuales sociales, o simplemente individuos sociales

En el estudio de la microeconomía se pone énfasis en el conocimiento de la teoría neoclásica, donde se plantea como supuesto básico la existencia de la competencia perfecta, es decir un mercado cuya estructura competitiva es satisfecha por cuatro condiciones, siendo una de ellas que cada consumidor actúa racionalmente con el fin de maximizar su bienestar. Es decir que las decisiones que tomamos, siempre son racionales, ese concepto sigue en la actualidad como base fundamental, pero a inicios del siglo XXI se ha ampliado mediante los estudios de la economía conductual y posteriormente con los avances en la neurociencia, haciendo que los libros de microeconomía sin renunciar a la teoría neoclásica, incorporen nuevos conceptos como es la intervención de las emociones en la toma de decisiones, al respecto Pindyck y Rubinfeld (2009, p. 77) nos dice que:

Sabemos que los consumidores no siempre deciden sus compras racionalmente. Por ejemplo, a veces compran impulsivamente, teniendo poco o nada en cuenta sus restricciones presupuestarias (y endeudándose como consecuencia). A veces no están seguros de lo que prefieren o se dejan influir por las decisiones de consumo de amigos y vecinos o incluso por los cambios de humor. Y aunque se comporten racionalmente, no siempre pueden tener totalmente en cuenta la multitud de precios y opciones a los que se enfrentan diariamente. Los economistas han desarrollado recientemente algunos modelos de la conducta de los consumidores que se basan en supuestos más realistas sobre la racionalidad y la toma de decisiones.

Al respecto existen varias teorías, donde también se desarrollan muchas que son poco serias y sin embargo sus publicaciones han tenido éxito en ventas, en ese sentido es nuestro deber profesional contribuir al esclarecimiento de las confusiones o inexactitudes que se dan en torno a este tema. De ahí que pretendemos analizar las principales teorías actuales a fin de contribuir al esclarecimiento de este tema tan polémico e importante.

Finalmente pretendemos avanzar en proponer la posibilidad de la relación de ambos conceptos (intuición y decisiones económicas) debido a que existen algunos indicios de vinculación a fin que se pueda abrir un debate interesante y polémico. 


\section{Conceptos sobre la intuición y como es entendido}

Unos de los conceptos más controvertidos y sobre el cual no existe aún, siquiera un posible acuerdo es el de la intuición, sin embargo, ha sido abordado desde varias aristas y a lo largo del proceso de desarrollo del pensamiento humano. Por ejemplo, la Real academia de la lengua española lo define por ejemplo como la facultad de comprender las cosas instantáneamente, sin necesidad de razonamiento.

Según el glosario de filosofía, el concepto de intuición procede del latín "intuitus" (imagen, mirada). Término con el que se designa, en general, la percepción directa e inmediata de un objeto y de sus relaciones, por parte del sujeto cognoscente. En tal percepción no puede haber ningún elemento intermedio, como podría ser el conocimiento discursivo o razonamiento, al que la intuición se opone radicalmente. La intuición puede ser sensible o inteligible, en función del tipo de objeto que se percibe.

Uno de los más grandes filósofos de la historia como es Platón, consideraba que el conocimiento por los sentidos es imperfecto y define cuatro formas de conocer, por la conjetura (Eicasia), creencia (Pístis), ambas están apegadas a lo sensible, discurso racional (Diánoia) que capta el ente matemático, y la Intuición (Nóesis) que la considera como la forma suprema de la inteligencia humana. Considera la intuición como un elemento innato que deviene del hombre, que no se desarrolla por experiencias anteriores y sería el único modo legítimo de conocer la realidad en toda su plenitud y pureza, únicamente por la intuición, el hombre puede llegar a conocer la verdadera realidad, el mundo inteligible de las ideas eternas.

Sobre este tema Echegoyen (1996, Vol.2) menciona que:

Descartes, considera la intuición como un acto de la mente por el cual vemos de forma inmediata, con claridad y distinción, la verdad de una proposición. Considera la intuición como: concepción que nace o tiene su origen en las "solas luces de la razón" y se acepta porque la mente ve inmediatamente su verdad. Considera que los dos actos de nuestra inteligencia o razón gracias a los cuales podemos llegar al conocimiento cierto son dos, la intuición y la deducción.

Según ello podríamos decir que Descartes concibe a la intuición en forma racional, como un elemento mediante el cual se pueden llegar a la conciencia, es decir a sus componentes de la conciencia pura, que vendrían 
a ser lo que se considera como las ideas innatas, es decir las verdades que se encuentran propiamente en el espíritu.

Al respecto Nakano (2008, p. 152) realiza un artículo interesante sobre Kant, y nos dice:

Kant afirma que la intuición formal presupone una síntesis, aunque precede a cualquier concepto.

Esta síntesis no se confunde con una determinación objetiva. La intuición formal no es la condición del pensar o del juzgar lo dado sensible, sino la condición de acuerdo con la cual la intuición empírica se da por primera vez. Entonces, no es adecuado presuponer la disyuntiva: la intuición o carece por completo de la articulación conceptual o es determinada objetivamente por las categorías. Con la noción de la intuición formal, Kant deja ver la necesidad de una articulación conceptual exactamente en el orden en el que la intuición es dada.

Así mismo, Castillo (2012, p. 1) respecto a Kant nos dice:

El único entendimiento humano tiene dos modos de ejercicio que son la inteligencia y la razón. La inteligencia conoce mediante evidencia, mostración o intuición intelectual, que es un conocimiento inmediato y directo. La razón conoce mediante demostración-deducción-inferencia, que es un conocimiento indirecto y mediato. Conocemos por intuición intelectual la esencia de las cosas sensibles y los primeros conceptos y principios del ser. Específicamente conocemos por intuición intelectual los primeros principios filosóficos, morales y matemáticos. La intuición empírica es el principio de toda demostración empírica y la intuición intelectual es el principio de toda demostración racional.

Una concepción dialéctica y no biologista como es la de Ortiz (2004), sugiere que es necesario diferenciar entre la estructura cognitiva como componente de la conciencia y el procesamiento cognitivo como parte de la actividad epiconsciente (percepción, imaginación, pensamiento y actuación personal) y entre los conocimientos como una clase de información psíquica consciente y conocimientos como una clase de información social. El sistema cognitivo productivo tiene el sistema de memoria en el neo córtex, que es la parte cerebral cuya aparición es la más reciente en la evolución de la especie humana y más específicamente en el neocórtex donde coinciden el lóbulo cerebral parietal, temporal y occipital. El sistema cognitivo consciente determina la organización de todo el conjunto de la conciencia en la forma de aptitudes y de la actividad epiconsciente en la forma de atención. Ejemplo: Las aptitudes cognitivas intuitivas y reflexivas. De este componente de la conciencia, depende la 
estructura del intelecto y por lo tanto determina las capacidades psíquicas de este componente del individuo. Así, aclara Ortiz (2004, p. 140) "así como las áreas afectivas tienen una disposición epigenética específica para guardar información afectiva, primero inconsciente y después consciente, así también debe ocurrir con las redes cognitivas que estamos estudiando".

Los humanos poseemos un sistema de recompensa cerebral que se activa ante la oportunidad de ganar algo, y parte de este sistema lo conforma la parte cerebral ubicada en la corteza prefrontal medial, que es la parte anterior de los lóbulos frontales del cerebro y dentro de ella más propiamente la parte cerebral ubicada en la corteza orbitofrontal, es decir en la parte cerebral sobre las orbitas que se ubican los ojos. Así mismo la corteza cingulada anterior (parte frontal del pliegue cerebral que parece como un collar alrededor de una estructura cerebral denominada cuerpo calloso). Entre ambas se encuentra las neuronas fusiformes que forman parte importante del proceso intuitivo. Al respecto Bermejo (2011, p. 178) "Las células fusiformes de la corteza cingulada anterior procesan e integran grandes volúmenes de información en muy poco espacio de tiempo y permiten responder a gran velocidad. Se trata pues de un sistema intuitivo cuya particularidad es la velocidad de la respuesta". Efectivamente existen varios tipos de neuronas, dentro de ellas existen las denominadas fusiformes, llamadas así, porque tienen una forma alargada como de ese instrumento utilizada para tejer llamada "Huso", este tipo de células ubicadas en la parte frontal del pliegue cerebral que parece como un collar alrededor de una estructura cerebral denominada cuerpo calloso, tienen la capacidad de procesar bastante información en poco tiempo y se presume que podría ser parte del sistema intuitivo.

Algunos investigadores consideran que la intuición es un producto no consciente, pero a su vez lo consideran dentro del plano cognitivo del ser humano, tal como lo concibe Fuster (2013, p.357) "Capacidad para adquirir conocimiento y obrar en consecuencia sin la conciencia ni el uso consciente de la razón". Efectivamente podemos considerarlo como una capacidad y en ese sentido correspondería al plano cognitivo, sin embargo ¿Cómo podría manifestarse sin ingresar al plano consciente? ¿Es que acaso es el inconsciente el que gobierna esta capacidad? Así mismo, manifiesta que no se relaciona con la razón, por ello estaría afirmando que es una capacidad no racional, lo que es contrario a los estudios experimentales, 
donde se demuestra que nuestro cerebro es un órgano preparado para discernir mediante atajos mentales, de otro modo el solo hecho de escoger, por ejemplo el color de camisa que vamos a ponernos, nos llevaría a decidir entre todo el vestuario de camisas que tenemos, por el contrario, nuestro cerebro fisiológicamente está organizado a simplificar cuando existe muchas opciones y reducirlas automáticamente a unas pocas sobre las cuales decide.

Es muy difundido y considerablemente aceptado por muchos la teoría del psicólogo israelí, Daniel Kahneman, premio nobel de economía 2002, sobre sus famosos procesos mentales de sistema tipo1 y tipo 2. Kahneman (2011) argumenta que la percepción y la intuición están muy relacionadas de tal modo que nunca ha sabido ver una separación clara entre una y otra. Para él hay dos familias principales de operación mental, donde el tipo 1 son fluidas y automáticas, con frecuencia inconscientes, son coherentes desde un punto de vista asociativo, a la que corresponde la percepción y la intuición, que no se pueden controlar y las del tipo 2 que se caracterizan por el control y el esfuerzo, por ser conscientes y lógicamente coherentes y se rige por reglas, son controladas, más lentas, más deliberadas. Estamos de acuerdo en que la intuición es fluida y automática, pero no podríamos confundirla con una percepción, es más bien una capacidad y la aísla de su proceso mental dos que es consciente, efectivamente podemos considerar a la intuición como un reflejo solo así podríamos aceptar que no es controlado, pero igualmente no hay otra manera que su manifestación se realice en el plano consciente para poder realizarse.

Pero al parecer la complejidad sobre la intuición es debido a que son resultado de varios procesos relacionados y tiene que ver básicamente con el conocimiento tácito que es distinto del conocimiento explícito. Al respecto Klein (2015, p.200) "La intuición tiene que ver con la experiencia práctica y con el saber o conocimiento tácito que es distinto del conocimiento explicito el que se refiere a datos objetivos".

Efectivamente todos los estudios recientes como el de Gary Klein parecen confirmar que la intuición forma parte del plano cognitivo y es un conocimiento tácito adquirido por la experiencia que si bien puede ser asimilada en forma espontánea e inconsciente, pero que al final de cuenta es integrada en el plano consciente, por ello podemos considerar 
que la intuición económica es la aptitud económica que forma parte de la estructura psíquica del intelecto, posibilitando anticiparse y notar en forma inmediata una situación económica determinada, producto del conocimiento implícito de la persona, que corresponde al sistema cognitivo y manifestado en el plano epiconsciente (percepción, imaginación, pensamiento y actuación personal) del individuo.

\section{Conceptos sobre la toma de decisiones y como es entendido}

La toma de decisiones es otro tema muy controversial debido a que hasta hace unas dos décadas la primacía la tenía la teoría neoliberal que ha primado desde fines del siglo XIX, ella sustenta la famosa teoría del homo economicus, es decir, considera que los seres humanos actuamos racionalmenteante decisiones económicas, analizamos toda lainformación disponible a nuestro alcance antes de tomar decisiones económica, para poder así, lograr alcanzar nuestros objetivos específicos al menor costo posible, es decir siempre estamos buscando analizar el costo-beneficio. Al respecto Mankiw, (2012, p. 18) menciona que:

Las lecciones fundamentales acerca de cómo las personas toman decisiones se resumen de la siguiente manera: las personas enfrentan diversas disyuntivas al buscar diferentes objetivos; los costos de cualquier acción se miden en términos de las oportunidades perdidas; las personas racionales toman sus decisiones considerando los beneficios y los costos marginales y modifican su comportamiento dependiendo de los incentivos.

La economía conductual ha profundizado el tema y una tendencia muy fuerte es la que desarrolla Arely (2008. P. 807) que menciona:

Todos nosotros somos mucho menos racionales en nuestros procesos de toma de decisiones de lo que presupone la teoría económica estándar. Nuestros comportamientos irracionales no son ni aleatorios ni insensatos; lejos de ello, son sistemáticos y previsibles. Todos cometemos los mismos tipos de errores una y otra vez, debido a la estructura básica de nuestro cerebro.

Sustenta la irracionalidad de nuestras decisiones y demuestra mediante experimentos que realizo en su mayoría con estudiantes universitarios del MIT como somos de predecibles los humanos.

Todo este contexto conlleva a pensar que somos seres más dados a las emociones que a la racionalidad, como si se diera por separado o antagónico. Somos seres con emociones, pero también somos seres 
pensantes porque todo se integra en la epiconsciencia, en opinión de Ortiz, (2004, P. 178) "La actividad epiconsciente viene a ser, entonces la actividad personal organizada psíquicamente desde el nivel de la conciencia. La actividad epiconsciente es, en sí misma, resultado de la activación e integración de la información psíquica guardada en las memorias neocorticales y paleocorticales del cerebro".

El psicólogo Alemán Gerd Gigerenzer director del Centro de Conducta Adaptativa y Cognición en el Instituto Max Planck para el Desarrollo Humano director del Centro Harding para la Alfabetización de Riesgos, en Berlín, Alemania, plantea que muchas personas toman decisiones por una sola razón dominante y de ahí define su propuesta sobre un nuevo término que él denomina heurística de reconocimiento (2015, p. 53) "Esta estrategia heurística solo presta atención a una variable, el ángulo de la mirada, sin necesidad de pensar en otras variables causales y puede lograr el mismo objetivo de una manera mucho más rápida y frugal y con menos probabilidades de fallo". Al respecto pone de ejemplo un estudio sobre como atrapan la pelota los expertos jugadores de béisbol, criquet o fútbol, sobre el cual concluye que por más que tengamos datos de la altura, fuerza, peso, viento, temperatura, etc. no podríamos concluir en atrapar la pelota porque no sigue una parábola exacta, ni un robot podría hacerlo debido a la cantidad de variables y al poco tiempo que existe para calcularlo, pero ¿Entonces como logran atrapar la pelota? Y concluye que la experiencia hace que estos jugadores ajusten la velocidad de la carrera de modo que el ángulo de su mirada entre el ojo y la pelota permanezca constante y solo así la pelota caerá justo donde está el jugador y podrá atraparla, es decir solo presta atención a una variable. Gigerenzen (2015, p. 54) "entendemos muy poco la relación entre teoría de la decisión y la teoría de la emoción y tampoco sabemos cómo desarrollamos un buen repertorio de elementos necesarios para tomar decisiones".

En la actualidad, pese a que en el campo de la neurociencia falta mucho que investigar, se está actualmente valiendo de un instrumento muy importante como es la resonancia magnética que son técnicas de imagen cerebral, mediante el cual podemos percibir cuales son las zonas cerebrales que se encienden a determinados estímulos. Ello ha dado origen a una nueva especialidad multidisciplinaria que es conocida 
como neuroeconomía que estudia la toma de decisiones económicas. Al respecto, Bermejo (2013, p. 51) menciona que:

El sistema de recompensa cerebral a través de la vía mesolimbocortical puede activar varias estructuras cerebrales, entre las que destacan el núcleo accumbens y la corteza prefrontal medial. El núcleo accumbens se estimula con la intensidad de la recompensa mientras que la corteza prefrontal medial se activa según las posibilidades de que obtengamos dicha recompensa. Veamos un ejemplo: si compramos un billete de lotería se activará en gran medida el núcleo accumbens, dado que existen muy pocas posibilidades de que nos toque y, en caso de hacerlo, tendríamos una gran recompensa. En cambio, si nos encontramos un billete de 5 euros en el suelo se estimulará muy poco el núcleo accumbens, dado que la recompensa no es muy alta, y se activará mucho la corteza prefrontal medial puesto que las posibilidades de que lo consigamos son muy grandes.

En ese sentido desde una visión básicamente biológica se establece la existencia de dos sistemas bien definidos, pero aun insuficientemente estudiados por la complejidad cerebral de nuestro organismo, el sistema de recompensa cerebral y el sistema de aversión al riesgo, sobre ellos refiere Bermejo, (2013, p. 46) "existen unas áreas cerebrales a favor de que realicemos tal acción o decisión, que conforman lo que se llama el sistema de recompensa y hay otras áreas cerebrales que están en contra de que tal acción o decisión se realice, que constituyen el llamado sistema de aversión al riesgo o aversión a la perdida. Del equilibrio resultante entre ambas fuerzas o sistemas dependerá la decisión final".

Esta idea es reforzada por Ortiz, (2004, p. 159), menciona que:

El neo córtex prefrontal dorso lateral es el sistema de memoria del componente conativo. Pacientes con lesiones prefrontales muestran una pérdida de su capacidad de iniciativa; son incapaces de decidir entre varias alternativas. La toma de decisiones está en base de las motivaciones, actitudes conativas y atributos psíquicos del carácter. "Se ha sugerido que el cerebro debe tener un mecanismo para decidir sin tener un cabal conocimiento tanto de las posibles alternativas, como del cálculo de probabilidades. Esto presupone que nuestro componente cognitivo no sería suficiente, aunque tal vez lo sea para las decisiones más rutinarias e intrascendentes.

Por lo expuesto no podemos ver por separado las tomas de decisiones de un aspecto biológico y de un aspecto psicológico o emocional, es necesario integrarlo en todo a la luz de una psicobiología moderna, que nos pueda dar mayor perspectiva de entendimiento, solo así podemos entender los resultados de los experimentos que se vienen estudiando 
en la neuroeconomía sustentada en una base del entendimiento de la personalidad humana, al respecto Ortiz, (2008, p. 23) menciona que:

La conciencia queda organizada ya no en dos sino en tres sistemas psíquicos: afectivoemotivo, cognitivo-productivo y conativo-volitivo; y sobre la base de las tres formas de información psíquica que cada una contiene, la personalidad misma será reestructurada en tres componentes: temperamento, intelecto y carácter, respectivamente. En otras palabras, la actividad consciente, sino reestructura cinéticamente no solo la actividad inconsciente, sino todos sus procesos neurales, metabólicos y celulares, hasta que la integridad del individuo quede reestructurada socialmente.

Las decisiones económicas del individuo en virtud de ello son producto de una integración de todos los elementos que resultan finalmente de su personalidad, podríamos por ello determinar que es la elección económica entre dos o más opciones cuyo resultado es desconocido para el individuo, procesada en el neocórtex prefrontal, correspondiendo a al sistema conativo volitivo que responden a sus motivaciones, intereses y actitudes, en base a su tradición, cultura y economía.

Es importante aclarar que la consideración de los economistas sobre la toma de decisiones no solo ha contemplado la racional y matematizada, al respecto Siller, (2011, p. 2), menciona: "John Maynard Keynes creía que la mayor parte de las decisiones económicas se producen en situaciones ambiguas donde las probabilidades son inciertas. Por eso concluyó que el ciclo económico depende en gran medida de la fluctuación de impulsos que denominó "espíritus animales": contenidos de la mente que los economistas no comprenden".

En la última década se ha desarrollado un nuevo campo interdisciplinario conocido como neuroeconomía que justamente estudia fundamentalmente la toma de decisiones, y aunque esta nueva disciplina esta recién en surgimiento, uno de los estudios más avanzado es el desarrollado por Pol Glimcher (2009, p.341), que menciona:

El reto capital que enfrentan los científicos neurales es relacionar el comportamiento con el cerebro. Parece claro que, para lograr ese objetivo, se requerirá una teoría del comportamiento .... parece natural suponer que una parte de los neuroecónomos desempeñará un papel crítico en explicar cómo resuelven realmente los cerebros de los seres humanos y otros animales los problemas de maximización que estas dos disciplinas han identificado".

Así mismo (2009, p. 356) manifiesta: "A fin de cuentas, la economía es una ciencia biológica. Es el estudio de cómo eligen los seres humanos. La 
elección es manifiestamente un proceso biológico. Entender verdaderamente cómo y porque los seres humanos eligen como lo hacen, requerirá sin duda una ciencia neuroeconómica". Hasta finales del siglo XX, aunque no existía un consenso en la definición de las ciencias económicas, existía la aceptación de considerar el estudio de la economía como una ciencia social, sin embargo en la actualidad, con el desarrollo de las neurociencias, las condiciones exigen profundizar las investigaciones de las ciencias económicas en la toma de decisiones y por ende investigar la intervención del cerebro en la toma de decisiones, así surge una nueva disciplina como es la neuroeconomía, ampliando así el panorama de las ciencias económicas y haciéndola ingresar al campo de las ciencias biológicas.

Glimcher, luego de un análisis extenso sobre la forma de la toma de decisiones y realización de varios experimentos, ha dado a entender que existe dos procesos, sistema de valoración y sistema de decisión, que están vinculadas, concibe que las emociones influencian en la toma de decisiones, pero niega la contradicción entre la racionalidad y las emociones. No considera que lo emocional está por un lado determinando valoraciones de los objetos y acciones y por otro lado lo racional. Para él ambos sistemas de valoración y decisión están íntimamente relacionados.

\section{Conclusiones}

La intuición económica no es irracional, es una aptitud que forma parte de la estructura psíquica del intelecto y por ende se manifiesta en el plano de la percepción, imaginación, pensamiento y actuación personal de la persona.

La decisión económica al ser procesada en el neocórtex pre frontal, que es la parte cerebral cuya aparición es la más reciente en la evolución de la especie humana, corresponde al sistema conativo volitivo, responde a las motivaciones, intereses y actitudes de la persona, en base a su tradición, cultura y economía. En ese sentido, si bien las emociones juegan un rol de influencia importante, no están apartadas de la parte racional, ni existe una exclusión entre ellas.

Así mismo la intuición y las decisiones son procesos que están íntimamente ligados, donde toda decisión es producto en alguna medida de la intuición, aunque ésta no siempre influye en ella necesariamente, mientras que no toda intuición conlleva a fin de cuentas a una decisión. 


\section{Bibliografía}

Ariely, Dan (2008). Las trampas del deseo. Como controlar los deseos que nos llevan al error. Spa. Pdf. Titivilluse Pub base r1.2, p. 921

Bermejo, P; Dorado, R; Zea-Sevilla, M y Sánchez, V. Neuroanatomía de las decisiones financieras. En: Revista Neurología. Vol. 26, Nº 3, p. 173-181.

Bermejo, Pedro y Izquierdo Ricardo (2013). Tu dinero y tu cerebro, Conecta; Barcelona, España, 191p.

Castillo Vegas, Juan (2012). La intuición intelectual en Kant. En: Do Direito UNISC, julio-diciembre, $\mathrm{N}^{\circ} 38$, p. 178-201.

Fuster, Joaquín (2013). Cerebro y libertad. Ariel; Barcelona, España, 375p.

Gigerenzer, Gerd (2015). Heuristica inteligente. John Brockman; Barcelona, España, $393 p$.

Glimcher, Paul (2009). Decisiones, incertidumbre y el cerebro. Fondo de cultura económica; Mexico, D. F. 389p.

Kahneman, D; Gigerenzen G; Klein G; Las mejores decisiones. John Brockman; Barcelona, España, 393p.

Kandel, E. Schwartz J. y Jessell T. Principios de neurociencia. Mcgraw Hill; Madrid, España, 1321p.

Lindstrom, M (2009). Compradicción. Verdades y mentiras de porque las personas compran. Norma; Bogotá, Colombia 248p.

Laza, Sebastián (2005). Neuroeconomía y teoría del consumidor. http://seblaza. blogspot.pe/2015/03/neuroeconomia-y-teoria-del-consumidor.html, p. 7

Maya, Guillermo (1993). La teoría neoclásica: reflexiones. En: Revista ensayos de economía de la universidad Nacional de Colombia. Medellin-Colombia; Vol. 27, No 50, p. 166.

Nakano, Hirotaka (2008). La distinción kantiana entre la forma de la intuición y la intuición formal. En: Revista signos filosóficos. México; Vol. 10, No 19, p. 152.

Ortiz, Pedro (1995). La afectividad humana. En: Revista Psiquiátrica Peruana. LimaPerú; Vol. 2, № 3, p.145-162.

Ortiz, Pedro (2004). Cuadernos de Psicobiología social. Fondo editorial de la UNMSM; Lima, Perú, 262p.

Ortiz, Pedro (2008). Educación y formación de la personalidad. Fondo editorial de la UCH; Lima, Perú, 310p. 
Ortiz, Pedro (2009). Introducción a una psicobiología del hombre. Fondo editorial de la UNMSM; Lima, Perú, 378p.

Pindyck y Rubinfeld (2009). Microeconomía. Pearson educación S. A.; Madrid, España, $850 \mathrm{p}$.

Ovejero, Felix (2005). La mente de la economía o la economía de la mente. https:// www.jstor.org/stable/30230652, p.16

Siller, Robert (2011). La revolución de la neuroeconomía.http://historico. prodavinci.com/2011/11/25/ciencia-y-tecnologia/la-revolucion-de-laneuroeconomia-por-robert-j-shiller/, p. 3 
LUIS ARTURo GUERra HEREDIA 